\title{
Collocation method applied to unsteady flow of gas through a porous medium
}

\author{
Subrahamanyam Upadhyay ${ }^{1 *}$, K. N. Rai ${ }^{2}$ \\ DST-CIMS, BHU, Varanasi, India \\ *Corresponding author E-mail: subabbu16@gmail.com
}

Copyright (C)2014 Upadhyay and Rai. This is an open access article distributed under the Creative Commons Attribution License, which permits unrestricted use, distribution, and reproduction in any medium, provided the original work is properly cited.

\begin{abstract}
In this article, we study a two point boundary value problem of non linear differential equation on a semi infinite domain that describes the unsteady flow of gas through a porous medium. Under special transform, we convert this problem to boundary value problem in compactly supported domain [0,1]. An algorithm provided for obtaining solution by Legendre wavelet collocation method. This method is effectively used to determine y (t) and its initial slope at the origin. The convergence and stability analysis is provided. The results thus obtained are compared with the those obtained from modified decomposition method [5], Variational iterational method [6], rational Chebyshev functions method (RCM) [7] and radial basis function (RBF) collocation method [10]. It has been observed that the proposed method provide better results with lesser computational complexity.
\end{abstract}

Keywords: Convergence and stability analysis, Legendre Wavelets, Legendre wavelet collocation method, Kidder's equation.

\section{Introduction}

The nonlinear partial differential equation that describes the unsteady flow of gas through a semi-infinite porous medium has been studied by H. T. Davis [1] in the form

$\nabla^{2} F^{2}=A^{2} \frac{\partial F}{\partial t}$

where $\mathrm{F}$ is the pressure within porous medium, $A$ is a constant and $\nabla^{2}$ is Laplace operator.

In term of dimensionless variable $\rho, \xi$, and $\tau$, the one dimensional problem from (1) come out to be

$\frac{\partial}{\partial \xi}\left(\rho \frac{\partial \rho}{\partial \xi}\right)=\frac{\partial \rho}{\partial \tau}$

Under transform

$\rho^{2}=1-\alpha, \alpha \in(0,1)$

$t=\frac{\xi}{2 \sqrt{\tau}}$ 
the nonlinear partial differential equation (2) reduces to the nonlinear ordinary differential equation

$y^{\prime \prime}(t)+2 t \frac{y^{\prime}(t)}{\sqrt[2]{1-\alpha y(t)}}=0$.

The boundary conditions required by the physical problem are as follows

$y(0)=1$,

$\lim _{t \rightarrow \infty} y(t)=0$

R. P. Agarval, D. Regan studied the existence of solution [2], upper and lower solution [3] of the problem (3) - (5). Kidder [4] solved the above problem (3) - (5), using perturbation technique. A perturbation series for the solution is as follows

$y(t)=\sum_{i=0}^{\infty} \alpha^{n} y^{(n)}(t)$

where $y^{(n)}(t)$ denotes nth derivative of $y(t)$ with respect to $x$ and the expainsion

$$
\sqrt[2]{1-\alpha y(t)}=1+\frac{\alpha y}{2}+\frac{3}{8} \alpha^{2} y^{2}+\ldots
$$

It is easily seen that the complexity of the calculations increases rapidly with increasing order of the terms [4]. Modified decomposition method [5] and Variational iteration method [6] have been used to solve problem (3) (5). After using these methods [5,6], Pade Approximation is utilized to convert polynomial approximation in to rational functions. K. Parand et al. [7, 8, 9, 10] solved problem (3) - (5) using generalized Laguerre polynomials and rational Chebyshev collocation method, Lagrangian method, modified generalized Laguerre functions Pseudo spectral method, RBF collocation method.

The solution of ordinary differential equations by Tau method and application of Chebyshev polynomial refers at least from time of Lenczos (1938) [11]. In the analytic study of differential equations, orthogonal function and polynomials have been used by Canuto et al [12] and A. Finlayson, L. E. Scriven [13]. If the solution function and coefficient functions are analytic on the close interval $[a, b]$, spectral methods are very efficient and suitable [12]. If at least one of the coefficient or solution function is not analytic on the close interval $[a, b]$, the spectral method does not work very well $[12,14,15]$. As described before, spectral (Collocation, Galerkin, Tau and Pseudo spectral) methods do not work well for this kind of problem. F. Mohammadi et al [16] used Galerkin method with Legendre wavelets, solved this problem with Dirichlet boundary conditions and obtained good results. To obtain solution of Legendre and Bessel differential equations, M. Razzaghi and S. Yousefi [17] used Legendre wavelets and its operational matrix of integration.

The Purpose of this paper is to solve equation (3) - (5), using collocation method with Legendre wavelet as a basis functions. The paper is organized in following section. In section2, Legendre wavelet and its operational matrix of integration are introduced. Present method and its convergence and stability analysis are provided in section 3. The results and discussion is given in section 4 . In section 5 we have concluding remarks.

\section{Wavelet}

Morlet (1982) first introduced the idea of wavelets as a family of functions constructed from translations and dilations of a single function called mother wavelet and defined by

$\psi_{s, r}(x)=|s|^{\frac{-1}{2}} \psi\left(\frac{x-r}{s}\right), s, r \in R, s \neq 0$

where $s$ is scaling parameter, $r$ is the translation parameter and $R$ denotes set of real numbers 


\subsection{Legendre wavelets}

The Legendre wavelets [16, 17], $\psi_{n, m}(x)=\psi(k, \hat{n}, m, x)$ we have four arguments

$k=1,2,3 \ldots$,

$n=1,2, \ldots, 2^{k-1}$,

$\hat{n}=2 n-1$,

$m$ is the order of Legendre polynomial and $x$ is the normalized time. They are defined on the closed interval $[0,1]$ by

$\psi_{n, m}(x)= \begin{cases}\sqrt{(m+1 / 2)} 2^{k / 2} P_{m}\left(2^{k} x-\hat{n}\right) & , \quad \frac{\hat{n}-1}{2^{k}} \leq x \leq \frac{\hat{n}+1}{2^{k}} \\ 0 & , \quad \text { otherwise. }\end{cases}$

Where the dilation parameter is $s=2^{-k}$ and the translation parameter is $r=\hat{n} 2^{-k} . P_{m}(x)$ is denoted by Legendre polynomial of order $m, m=0,1, \ldots, M-1$, which are orthogonal with respect to the weight function $w(x)=1$ on the interval $[-1,1]$, and satisfy the following recursive formula

$P_{0}(x)=1$,

$P_{1}(x)=x$,

$P_{m+1}(x)=\frac{2 m+1}{m+1} x P_{m}(x)-\frac{m}{m+1} P_{m-1}(x)$.

\subsection{Fourier approximation:}

A function defined in domain $[0,1]$ may be expressed as

$f(x)=\sum_{n=1}^{\infty} \sum_{m=0}^{\infty} c_{n, m} \psi_{n, m}(x)$,

where $c_{n, m}=<f(x), \psi_{n, m}(x)>$ in which $<,>$ denotes the inner product. If the infinite series in (6) is truncated, then it can be written as

$f(x) \approx \sum_{n=1}^{2^{k-1}} \sum_{m=0}^{M-1} c_{n, m} \psi_{n, m}(x)=C^{T} \psi(x)$

where $C$ and $\psi(x)$ are column matrices of order $2^{k-1} M \times 1$, given by

$C=\left[c_{10}, c_{11}, \ldots, c_{1 M-1}, c_{20}, c_{21}, \ldots, c_{2 M-1}, c_{2^{k-1} 0} c_{2^{k-1} 1}, \ldots, c_{2^{k-1} M-1}\right]^{T}$
$\psi(x)=\left[\psi_{10}(x), \psi_{11}(x), \ldots, \psi_{1 M-1}(x), \psi_{20}(x), \ldots, \psi_{2 M-1}(x), \ldots\right.$
$\left.\psi_{2^{k-1} 0}(x) \psi_{2^{k-1} 1}(x), \ldots, \psi_{2^{k-1} M-1}(x)\right]^{T}$.

\subsection{Operational matrix of integration}

The integration of the wavelets $\psi(x)$ can be obtained as

$\int_{0}^{x} \psi(s) d s=P \psi(x)$.

$P$ is $2^{k-1} M \times 2^{k-1} M$ operational matrix of integration $[16,17]$ defined by

$P=\frac{1}{2^{k}}\left(\begin{array}{ccccc}L & O & O & \cdots & O \\ 0 & L & O & \cdots & O \\ 0 & 0 & L & \cdots & O \\ \vdots & \vdots & \vdots & \ddots & \vdots \\ 0 & 0 & 0 & \cdots & O \\ 0 & 0 & 0 & \cdots & O\end{array}\right)$ 
where $F$ and $L$ are $M \times M$ matrices given by

$$
O=\left(\begin{array}{cccc}
2 & 0 & \cdots & 0 \\
0 & 0 & \cdots & 0 \\
\vdots & \vdots & \ddots & \vdots \\
0 & 0 & \cdots & 0
\end{array}\right)
$$

and

$$
L=\left(\begin{array}{cccccccc}
1 & \frac{1}{\sqrt{3}} & 0 & 0 & \cdots & 0 & 0 & 0 \\
\frac{-1}{\sqrt{3}} & 0 & \frac{1}{\sqrt{15}} & 0 & \cdots & 0 & 0 & 0 \\
0 & \frac{-1}{\sqrt{15}} & 0 & \frac{1}{\sqrt{35}} & \cdots & 0 & 0 & 0 \\
0 & 0 & \frac{-1}{\sqrt{35}} & 0 & \cdots & 0 & 0 & 0 \\
\vdots & \vdots & \vdots & \vdots & \ddots & \vdots & \vdots & \vdots \\
0 & 0 & 0 & 0 & \cdots & \frac{1}{\sqrt{(2 M-3)(2 M-1)}} & 0 & \frac{1}{\sqrt{(2 M-3)(2 M-5)}} \\
0 & 0 & 0 & 0 & \cdots & 0 & \frac{-1}{\sqrt{(2 M-1)(2 M-3)}} & 0
\end{array}\right) \text { respectively. }
$$

\section{Algorithm of obtaining solution by Legendre wavelet collocation method (LWCM $(M, k)$ ) for Kidder's equation}

The algorithm of obtaining solution by $\operatorname{LWCM}(M, k)$ for Kidder's equayion given below in following steps.

Step 1: The first step of algorithm is to transform independent variable $\mathrm{t}$ to a new variable $\mathrm{x}$ which is defined on valid range $[0,1]$ of shifted ortho-normal Legendre polynomial through

$x=\exp (-t)$

After substitution of (14) in (3) - (5), the equation (3) - (5) is transformed to a new form as

$x(\sqrt{1-\alpha y(x)}) y^{\prime \prime}(x)-\left(2 \log _{e}(1 / x)-\sqrt{1-\alpha y(x)}\right) y^{\prime}(x)=0, x \in(0,1)$,

$y(0)=0$,

$y(1)=1$.

Step 2: We assume that the unknown function $y^{\prime \prime}(x)$ is given

$y^{\prime \prime}(x)=C^{T} \psi(x)$,

where $C$ is an unknown vector and $\psi(x)$ is vector which is defined in (10) and (11). We integrate equation (18) two times from 0 to $x$ and using equation (12), we get

$y^{\prime}(x)=y^{\prime}(0)+C^{T} P \psi(x)$,

$y(x)=y^{\prime}(0) x+C^{T} P^{2} \psi(x)$.

From (17) and (20), we have

$y^{\prime}(0)=1-C^{T} P^{2} \psi(1)$.

From (19), (20) and (21), we have

$y^{\prime}(x)=1-C^{T} P^{2} \psi(1)+C^{T} P \psi(x)$, 
$y(x)=\left(1-C^{T} P^{2} \psi(1)\right) x+C^{T} P^{2} \psi(x)$.

Using (18), (22), and (23) in (15), we obtained residual $R(C, x)$ as a function of $C$ and $x$ as follows

$R(C, x)=x\left(\sqrt{1-\alpha\left(\left(1-C^{T} P^{2} \psi(1)\right) x+C^{T} P^{2} \psi(x)\right)}\right) C^{T} \psi(x)-$

$\left(2 \log _{e}(1 / x)-\left(\sqrt{1-\alpha\left(\left(1-C^{T} P^{2} \psi(1)\right) x+C^{T} P^{2} \psi(x)\right.}\right)\right)\left(1-C^{T} P^{2} \psi(1)+C^{T} P \psi(x)\right)$

\section{Step 3:}

\section{1. $\quad$ LWCM $(M, k)$}

In collocation method, we choose $2^{k-1} M$ collocation points as

$x_{10}, x_{11}, \ldots, x_{1 M-1}, x_{20}, x_{21}, \ldots, x_{2 M-1}, x_{2^{k-1} 0} x_{2^{k-1} 1}, \ldots, x_{2^{k-1} M-1}$ in the open interval $(0,1)$ with

$R\left(C, x_{n, m}\right)=0, m=0,1,2, \ldots M-1, n=1,2, \ldots, 2^{k-1}$.

Step 4: The equations (24 represent systems of non linear equation for unknown vector $C$. The equation (24) can be solved for vector $C$. Using Newton Raphson method, the systems can be solved for vector $C$. After finding the unknown vector $C$, we can get the approximate solution of (15) - (17) by inserting $C$ into (23) .

Step 5: Using inversion of transform (14) in obtained solution of (15) - (17), we have required solution.

\subsection{Convergence Analysis}

To compute approximation error, we introduce an orthonormal basis of $L_{2}(R)$, with $2^{k-1} M$ vectors $\left\{\psi_{n, m}(x)\right\}$ defined in section 2.2 is an orthonormal basis of the some approximation space $V_{2^{k-1} M}$. Approximation error of linear sampling processes is related to the error of linear approximation in an orthonormal basis. These errors are computed from the decay of signal coefficients in this basis.

Theorem 3.2.1 Let $y(x)$ is real valued function defined on $[0,1]$ and its $m t h$ derivative $y^{(m)}(x)$ is bounded on $[0,1]$. The following inequality holds

where

$$
e(m, y) \leq \frac{K}{m !},
$$

$$
\begin{gathered}
e(m, y)=\left\|y(x)-y_{m}(x)\right\|_{L_{2}[0,1]}, \\
K=\sup \left\{y^{(m)}(x): x \in[0,1]\right\},
\end{gathered}
$$

and $y_{m}(x)$ denotes the approximate solution of initial value problem (15) - (17).

Proof Equation (23) is representing approximate solution of boundary value problem (15) - (17). It can be written in the form

$y_{m}(x)=\sum_{n=1}^{2^{k-1}} \sum_{m=0}^{M-1} d_{n, m} \psi_{n, m}(x)$,

with restriction that $y_{m}(x)$ is a polynomial of a degree $m$ that approximate $y^{*}(x)$ with minimum mean square error, where $\left\{\psi_{n, m}(x)\right\}$ is an orthonormal basis of the approximation space $V_{2^{k-1} M}$ of $L_{2}(R)$.

$$
\begin{aligned}
& \left\|y(x)-\sum_{n=1}^{2^{k-1}} \sum_{m=0}^{M-1} d_{n, m} \psi_{n, m}(x)\right\|_{L_{2}[0,1]}^{2} \leq \int_{0}^{1}\left[y(x)-y^{*}(x)\right]^{2} d x \\
& \int_{0}^{1}\left[y(x)-y^{*}(x)\right]^{2} d x \leq \int_{0}^{1}\left[\frac{1}{m ! 2^{m k}} \operatorname{Sup}\left\{y^{(m)}(x): x \in[0,1]\right\}\right]^{2} d x \\
& \leq\left[\frac{K}{m ! 2^{m k}}\right]^{2}
\end{aligned}
$$


where $y^{*}(x)$ is interpolating polynomials of $\mathrm{y}(\mathrm{x})$ and we have used well known maximum error bound for interpolation.

$\leq\left[\frac{K}{m !}\right]^{2}$, for $k>0, m \geq 0$.

From (25) and (26), we have required result.

$e(m, y) \leq \frac{K}{m !}$

Equation (27), shows that $e(m, y)$ approaches to zero as $m$ tend to infinity.

Theorem 3.2.2 Let $E(x)$ and $y_{m}(x)$ be real valued function lies in $L_{2}[0,1]$ and $<y_{m}(x)+E(x), y_{m}(x)+E(x)>$ exist, where

$L_{2}[0,1]=\left\{f:[0,1] \rightarrow C: \int_{0}^{1}|f(x)|^{2} d x<\infty\right\}$ space,

$y_{m}(x)$ is an approximate solution of boundary value problem (15) - (17) given in equation (23). Then $D=-1+C^{T} P^{2} \psi(1)-C^{T} P^{2} d$.

whenever $D=\sup \{E(x): x \in[0,1]\}$ and $\psi(x)$ is defined (11), $d$ denotes column matrix of order $2^{k-1} M \times 1$. The first entry of matrix $d$ is one and remaining zeros.

Proof Since

$<y_{m}(x)+E(x), y_{m}(x)+E(x)>=\int_{0}^{1} y_{m}^{2}(x) d x+2 \int_{0}^{1} y_{m}(x) E(x) d x+\int_{0}^{1} E^{2}(x) d x$.

We have,

$D^{2}+2 D \int_{0}^{1} y_{m}(x) d x+\int_{0}^{1} y_{m}^{2}(x) d x \geq 0$,

then there is a constant $K \geq 0$ such that

$D^{2}+2 D \int_{0}^{1} y_{m}(x) d x+\int_{0}^{1} y_{m}^{2}(t) d x-K=0$.

The root of above quadratic equation in $D$ is

$D=-\int_{0}^{1} y_{m}(x) d x \pm \sqrt{\left(\int_{0}^{1} y_{m}(x) d x\right)^{2}-\int_{0}^{1} y_{m}^{2}(x) d x+K}$.

Since D is unique, then D will be

$D=-\int_{0}^{1} y_{m}(x) d x$

when

$\left.K=-\int_{0}^{1} y_{m}(x) d x\right)^{2}+\int_{0}^{1} y_{m}^{2}(x) d x$

Obtained unique D and from equation (23), we get

$D=-1+C^{T} P^{2} \psi(1)-C^{T} P^{2} d$. 
Table 1: Exhibited the value $y(t)$ at $\alpha=0.5$.

\begin{tabular}{rrrrcr}
\hline$x$ & $y$ Kidder & $y(2 / 2)][5,6]$ & $y(3 / 3)[5,6]$ & $R C F[7]$ & $y \operatorname{LWCM}(9,1)$ \\
\hline 0.1 & 0.8816588283 & 0.8633060641 & 0.8979167028 & 0.88042558 & 0.8814752903 \\
0.2 & 0.7663076781 & 0.7301262261 & 0.7985228199 & 0.76963630 & 0.7666110186 \\
0.3 & 0.6565379995 & 0.6033054140 & 0.7041129703 & 0.66402932 & 0.6572701887 \\
0.4 & 0.5544024032 & 0.4848898717 & 0.6165037901 & 0.55870984 & 0.5556775254 \\
0.5 & 0.4613650295 & 0.3761603869 & 0.5370533796 & 0.46068185 & 0.4632588205 \\
0.6 & 0.3783109315 & 0.2777311628 & 0.4665625669 & 0.37504968 & 0.3807807670 \\
0.7 & 0.3055976546 & 0.1896843371 & 0.4062426033 & 0.30320332 & 0.3085393048 \\
0.8 & 0.2431325473 & 0.1117105165 & 0.3560801699 & 0.24431600 & 0.2464487274 \\
0.9 & 0.1904623681 & 0.0432367323 & 0.3179966614 & 0.19666414 & 0.1940914931 \\
1 & 0.1587689826 & 0.0164675084 & 0.2900255005 & 0.15835106 & 0.1587755564 \\
\hline
\end{tabular}

Table 2: Exhibited the value $y^{\prime}(0)$.

\begin{tabular}{rrrrr}
\hline$\alpha$ & $y(2 / 2)[5,6]$ & $y(3 / 3)[5,6]$ & $R C F[7]$ & $y \operatorname{LWCM}(9,1)$ \\
\hline 0.2 & -2.441894334 & -1.786475516 & Not calculated & -2.121683561 \\
0.5 & -1.373178096 & -1.025529704 & -1.26259357 & -1.199258245 \\
0.8 & -0.863340021 & -0.477669728 & Not calculated & -0.690065816 \\
\hline
\end{tabular}

\section{Results and discussion}

Kidder's equation is a highly non linear differential equation with semi infinite boundary condition. Because of this its solution is very difficult task. The solution of Kidder's equation is important because, it describe complex physical phenomenon of the unsteady flow of gas through a porous medium. An algorithm of present method provided in section 3. The steps of algorithm are easy to understand and implementable on MATLAB. From Theorem 3.2.1, we observe that $x_{m}$ tend to $x$ and $e(m, y)$ tend to zero as $m$ tend to infinity. In this results we say that error can be minimize by taking large number of Legendre wavelet basis functions. From theorem 3.2.2, we observe that maximum error of solution on the closed interval $[0,1]$ obtained by $\operatorname{LWCM}(M, k)$ is depending on the column vector $C$. The elements of column vector $C$ are the coefficients of Legendre wavelet series expansion because of this the maximum error of solution on the closed interval $[0,1]$ is bounded.

In the physical observation of the unsteady gas problem, $y^{\prime}(0)$ has an important issue [1]. The solution $y(t)$ and its slope at origin $y^{\prime}(0)$ obtained by $\operatorname{LWCM}(9,1)$ and those obtained by modified decomposition $(y(2 / 2)$, $y(3 / 3))$ [5], Variational iteration $(y(2 / 2), y(3 / 3))[6]$ and RCF [7] methods given in Table 1 - 2. In solution $\mathrm{y}(\mathrm{t})$ of Kidder's equation by modified decomposition [5] and Variational iteration [6] methods containing its slope at origin as a unknown constant. For removing this constant, they used Pade approximation because of this, these method preserved large truncation errors and computations are very lengthy in comparison to present method. In RCF [7] method, the solution also preserve large truncation error than present method. The comparative study of solution by $\operatorname{LWCM}(9,1)$, shooting Ronge kutta [10] method and RBF [10] method given in Fig. 1. Shooting Ronge kutta method have large truncation error because of this present method provide better results than shooting Ronge kutta [10] method and RBF [10].

\section{Conclusion}

The present method is easy to understand, no any complexity in computations and easy to implementable on MATLAB. From Theorem 3.2.1 and 3.2.2; we conclude that $L_{2}$ norm of error tends to zero as $m$ tend to infinity and maximum error on the closed interval $[0,1]$ is bounded The present method provided the physical observation of the unsteady gas problem. From section 4, we conclude that the results obtained by present method provided better accuracy than those obtained by others methods. The method can also used to highly non linear singular problem. 


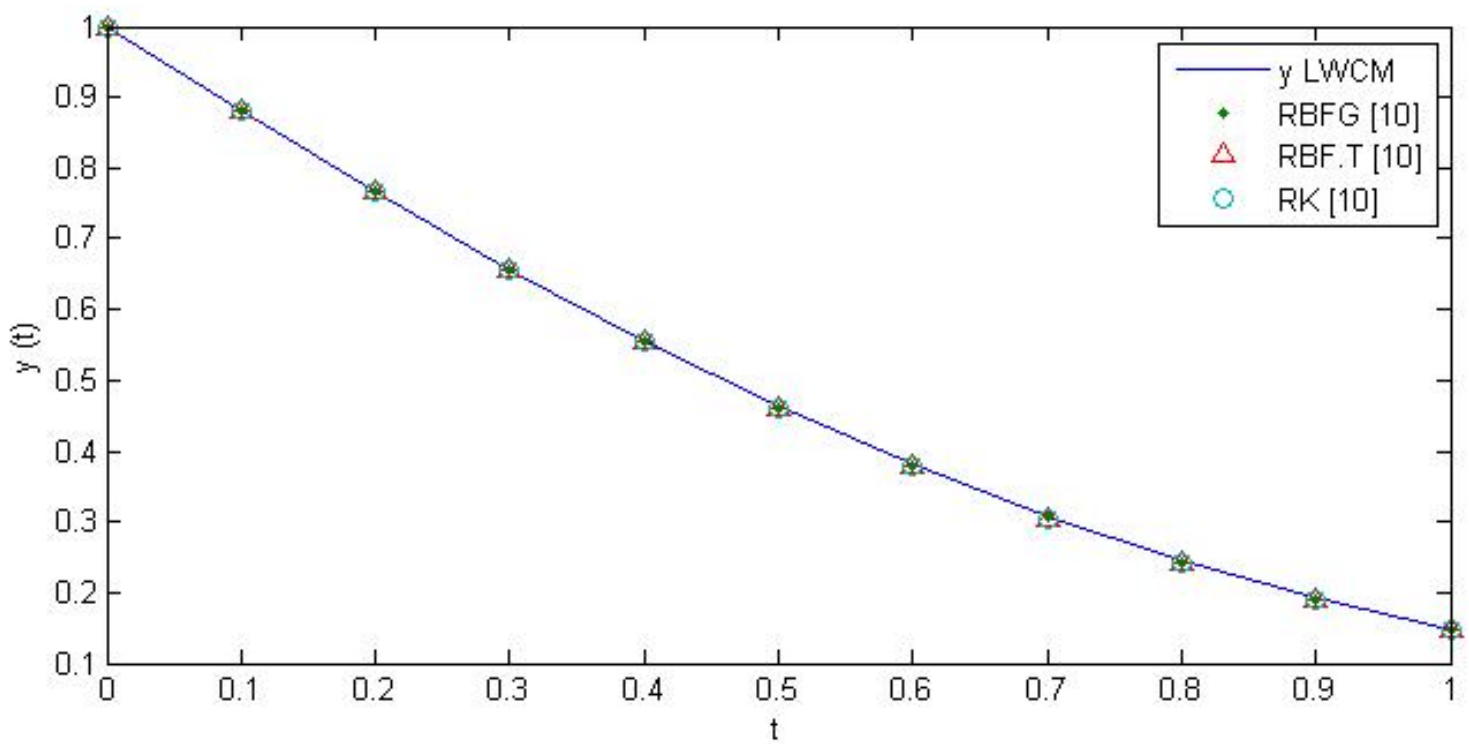

Fig.1: Comarative study of solution of Kidder's equation.

\section{References}

[1] H.T. Davis, Introduction to Nonlinear Differential and Integral Equations, Dover Publications, New York, 1962.

[2] R. P. Agarval, D. Regan, Infinite interval problems for differential, diifference and integral Equations, Springer Science-Business Media, 2001.

[3] R. P. Agarval, D. Regan, Non-linear boundary value problems on the semi infinite interal, all upper and lower solution approach, MATHHMATIKA, 49 (2002), 129-140.

[4] R.E. Kidder, Unsteady flow of gas through a semi-infinite porous medium, J. Appl. Mech. 27(1957) 329332.

[5] A. M. Wazwaz, The modified decomposition method applied to unsteady flow of gas through a porous medium, Appl. Math. Comput. 118 (2001) 123-132.

[6] M. A. Noor, S. T. Mohyud-Din, Variational iteration method for unsteady flow of gas through a porous medium using He's polynomials and Pade approximants, Computers and Mathematics with Applications 58 (2009) 2182-2189.

[7] K. Parand, M. Shahini and A. Taghavi, Generalized Laguerre Polynomials and Rational Chebyshev Collocation Method for Solving Unsteady Gas Equation, Int. J. Contemp. Math. Sciences, 4 (2009) 1005- 1011.

[8] A. Taghavi, K.Parand, and H. Fani, Lagrangian method for solving unsteady gas equation, World Academy of Science, Engineering and Technology35 (2009) 1016-1020.

[9] K. Parand, A. Taghavi, M. Shahini, Comparison between Rational Chebyshev and Modified generalized Laguerre Functions Psuedospectral method for solving LaneEmden and unsteady gas equation, ACTA PHYSICA POLONICA B, 40 (2009) 1749-1763.

[10] S. Kazem, J. A. Rad, K. Parand, M. Shaban, and H. Saberi, The numerical study on the unsteady flow of gas in a semi-infinite porous medium using an RBF collocation, International Journal of Computer Mathematics, 89 (2012) 22402258.

[11] C. Lanczos, Trigonometric interpolation of empirical and analytical functions, Journal of Mathematics and Physics 17 (1938) 123-129. 
[12] C. Canuto, M. Hussaini, A. Quarteroni, and T. Zang, Spectral Methods in Fluid Dynamics, Berlin Springer 1988.

[13] A. Finlayson, L.E. Scriven, The method of weighted residuals: a review, Applied Mechanics Reviews. 19 (1966) 735-748.

[14] B. Fornberg, A Practical Guide to Pseudospectral Methods, Cambridge, Cambridge University Press 1996.

[15] E. Babolian, M.M. Hosseini, A modified spectral method for numerical solution of ordinary differential equations with non-analytic solution, Applied Mathematics and Computation. 132 (2002) 341-351.

[16] F. Mohammadi, M.M. Hosseini and S. T. Mohyud-Din, Legendre wavelet galerkin method for solving ordinary differential equations with non-analytic solution, Int. J. of Sys. 42 (2011) 579-585.

[17] M. Razzaghi, S. Yousefi, Legendre wavelets operational matrix of integration, International Journal of Systems Sci. 32 (2001) 495-502. 